Annuaire suisse de politique de développement

25-2 | 2006

Paix et sécurité : les défis lancés à la coopération internationale

\title{
Coopération internationale entre paix et sécurité : portes d'entrée sur Internet
}

Xavier Tschumi Canosa et Thania Paffenholz

\section{(2) OpenEdition}

\section{Journals}

Édition électronique

URL : http://journals.openedition.org/aspd/282

DOI : $10.4000 /$ aspd.282

ISSN : 1663-9669

Éditeur

Institut de hautes études internationales et du développement

Édition imprimée

Date de publication : 1 octobre 2006

Pagination : 229-231

ISBN : 2-88247-064-9

ISSN : $1660-5934$

Référence électronique

Xavier Tschumi Canosa et Thania Paffenholz, « Coopération internationale entre paix et sécurité portes d'entrée sur Internet », Annuaire suisse de politique de développement [En ligne], 25-2 | 2006, mis en ligne le 26 janvier 2010, consulté le 08 septembre 2020. URL : http://journals.openedition.org/ aspd/282 ; DOI : https://doi.org/10.4000/aspd.282

Ce document a été généré automatiquement le 8 septembre 2020.

(c) The Graduate Institute I Geneva 


\title{
Coopération internationale entre paix et sécurité : portes d'entrée sur Internet
}

\author{
Xavier Tschumi Canosa et Thania Paffenholz
}

\section{Introduction}

1 Il suffit de se plonger dans les notes bibliographiques des articles composant le présent dossier pour se rendre compte de la richesse des sources qui alimentent la réflexion sur la thématique de la coopération internationale, entre paix et sécurité: cette thématique n'intéresse pas seulement les chercheurs du milieu académique, mais également les praticiens gouvernementaux, non gouvernementaux ou des organisations internationales.

2 Cette partie de l'ouvrage, qui se veut un point d'appui au lecteur désirant approfondir ses connaissances des domaines abordés dans le dossier, se fonde sur la présentation d'un certain nombre de sites Internet choisis. Elle ne fournit donc pas une liste exhaustive des ressources traitant de la thématique, tant elles sont nombreuses. En effet, il nous a paru plus utile que le lecteur puisse bénéficier de quelques commentaires descriptifs du contenu des sites retenus. De plus, ceux-ci constituent des portails offrant des liens vers d'autres sites d'intérêt.

\section{Focalisation du point d'appui}

3 La thématique du dossier trouve la grande majorité de ses sources en langue anglaise et ce point d'appui en présente certaines. Toutefois, nous avons décidé de présenter autant que possible des sites Internet proposant des contenus en langue française et/ou allemande, pour répondre aux besoins des lecteurs du présent dossier de l'Annuaire.

4 Les sites Internet gouvernementaux constituent évidemment des ressources essentielles pour le lecteur intéressé aux politiques de paix, de sécurité et de 
développement qui sont déployées par les Etats. Ils ouvrent des portes sur leur perception de ces notions, sur la base légale de la définition et de la mise en œuvre des politiques, et sur les opérations mêmes, y compris leurs moyens et leur évaluation. Nous n'entrerons pas plus dans le détail ici, les sites gouvernementaux étant faciles à trouver et les moteurs de recherche au sein de ces sites suffisamment développés pour permettre au lecteur l'accès à ses centres d'intérêt.

\section{Sites Internet consacrés aux conflits et à la paix}

Les instituts et centres de recherche qui travaillent sur les conflits et la paix existent dans pratiquement tous les pays du Comité d'aide au développement (CAD) de l'Organisation de coopération et de développement économiques (OCDE). Parmi ceux-ci, il faut mentionner les quelques instituts indépendants et internationaux suivants, bien qu'ils soient ancrés dans leurs pays respectifs :

\section{Department of Peace and Conflict Research, Uppsala University}

\section{<http://www.pcr.uu.se>}

Ce département universitaire a été établi en 1971 et, outre sa vocation de recherche, il offre des cours et des enseignements en matière de paix et de conflit. Le site Internet, en anglais, offre l'accès à toutes les recherches en cours ainsi qu'aux banques de données qui sont alimentées par ces programmes et projets de recherche.

\section{University of Colorado Conflict Research Consortium}

$7 \quad<$ http://conflict.colorado.edu>

Ce centre multidisciplinaire de recherche et d'enseignement sur les conflits et leur transformation a été établi en 1988. L'adresse Internet qui est indiquée ici est un portail ouvert sur les sites Internet du consortium, soit des banques de données, des sources d'informations ou des programmes d'enseignement en ligne. Toutes les ressources sont en anglais.

\section{Berghof Research Center for Constructive Conflict Management}

\section{$<$ http://www.berghof-center.org>}

Ce centre de recherche, établi à Berlin, a été fondé en 1993 dans le but d'explorer les procédures et modèles qui pourraient faciliter, d'une manière constructive, la résolution des conflits ethnopolitiques. Le site Internet, en anglais ou en allemand, donne accès aux deux piliers du centre de recherche, qui se situent à mi-chemin entre la théorie et la pratique :

\section{Berghof Handbook for Conflict Transformation}

$9 \quad<$ http://www.berghof-handbook.net>

Ce site constitue un recueil de ressources issues des connaissances, des expériences et des leçons tirées du travail de terrain des experts actifs dans la transformation des conflits armés. Il contient, d'une part, des articles sur la pratique de ces experts et sur 
la recherche qui y est consacrée et, d'autre part, une série de dialogues et de débats entre les praticiens et les chercheurs sur le thème de la transformation des conflits. Toutes les ressources sont en anglais mais certaines sont traduites dans d'autres langues (voir $>$ Translations).

\section{Berghof Foundation for Peace Support}

\section{<http://www.berghof-peacesupport.org>}

La mission de cette fondation, créée en 2004, est de promouvoir des approches systémiques permettant de prévenir et de transformer des conflits violents ainsi que de soutenir les processus de paix par la mise en place de réseaux d'organisations et d'initiatives. Tout ce qui touche à ces approches systémiques et aux projets de la fondation est accessible sur ce site. La majorité des documents sont en anglais mais il y en a également un certain nombre en allemand.

\section{International Crisis Group (ICG)}

11 <http://www.icg.org>

L'ICG est une organisation non gouvernementale indépendante dont les membres sont répartis dans les pays en conflit ou à proximité. A partir des observations qu'ils font sur le terrain, ces experts produisent des rapports et des recommandations destinés aux décideurs politiques au niveau international. Ces rapports sont disponibles sur le site Internet. Les ressources sont organisées par pays ou région, mais aussi par thème. Le site est le plus complet en anglais. En français, seule une partie des ressources est accessible, alors qu'il n'y a aucune ressource en allemand.

Parmi les sites Internet de centres suisses dédiés aux questions de paix et de sécurité, voici notre sélection :

\section{Center for Security Studies (css)}

13 <http://www.css.ethz.ch>

Ce centre de recherche et d'enseignement fait partie de l'Ecole polytechnique fédérale de Zurich (EPFZ). Il a été créé en 1986 et se concentre sur l'étude des politiques de sécurité. Le site Internet du css donne accès, en allemand ou en anglais, aux recherches menées dans les domaines de la politique internationale de sécurité et de la politique suisse de sécurité, y compris aux publications qui y sont liées. Le css abrite et gère également, sur mandat de la Confédération suisse, le réseau des relations internationales et de la sécurité décrit ci-dessous :

\section{International Relations and Security Network (ISN)}

<http://www.isn.ethz.ch>

Ce réseau a été établi en 1994 et reçoit son financement du Département fédéral de la défense, de la protection de la population et des sports (DDPS) et de l'EPFZ. Il offre des services d'information, d'analyse et de formation sur les questions de sécurité. Le site Internet, uniquement en anglais, donne accès à toutes les recherches et publications du 
réseau, à ses ressources de formation et d'éducation (e-learning en particulier) ainsi qu'à ses services de nouvelles.

\section{Center for Peacebuilding (KоFF)}

\section{$<$ http://www.swisspeace.org/koff>}

Ce centre est pensé comme un trait d'union entre l'administration fédérale et les organisations non gouvernementales suisses actives dans le domaine de la consolidation de la paix. Son but est de veiller à ce que la politique de paix de la Suisse dans son ensemble conserve une certaine cohérence, tant dans sa base théorique que dans ses activités opérationnelles. Le site Internet contient des analyses et des programmes de formation dans le domaine, par pays et par thème. Il donne également accès aux sites Internet des 45 oNG qui sont rattachées au centre. Les ressources sont en anglais principalement, mais certains documents existent aussi en français et en allemand. 